\author{
Transmission Electron Microscopy Investigation \\ of Texture Development in Magnesium Oxide Buffer Layers* \\ R. E. Koritala, M. P. Chudzik, and U. Balachandran \\ Energy Technology Division \\ Argonne National Laboratory \\ Argonne, IL 60439 \\ Z. P. Luo and D. J. Miller \\ Materials Science Division \\ Argonne National Laboratory \\ Argonne, IL 60439 \\ and \\ C. R. Kannewurf \\ Department of Electrical Engineering \\ Northwestern University \\ Evanston, IL 60208
}

September 2000

The submitted manuscript has been created by the
University of Chicago as Operator of Argonne
National Laboratory ("Argonne") under Contract
No. W-31-109-ENG-38 with the U.S. Department of
Energy. The U.S. Government retains for itself, and
others acting on its behalf, a paid-up, nonexclusive,
irrevocable worldwide license in said article to
reproduce, prepare derivative works, distribute
copies to the public, and perform publicly and
display publicly, by or on behalf of the Government.

Paper to be submitted to the Applied Superconductivity Conference, ASC 2000, Virginia Beach, VA, September 17-22, 2000.

*Work supported by the U.S. Department of Energy (DOE), Office of Energy Efficiency and Renewable Energy, as part of a DOE program to develop electric power technology, under Contract W-31-109-Eng-38. 


\section{DISCLAIMER}

This report was prepared as an account of work sponsored by an agency of the United States Government. Neither the United States Government nor any agency thereof, nor any of their employees, make any warranty, express or implied, or assumes any legal liability or responsibility for the accuracy, completeness, or usefulness of any information, apparatus, product, or process disclosed, or represents that its use would not infringe privately owned rights. Reference herein to any specific commercial product, process, or service by trade name, trademark, manufacturer, or otherwise does not necessarily constitute or imply its endorsement, recommendation, or favoring by the United States Government or any agency thereof. The views and opinions of authors expressed herein do not necessarily state or reflect those of the United States Government or any agency thereof. 


\section{DISCLAIMER}

Portions of this document may be illegible in electronic image products. Images are produced from the best available original document. 


\title{
Transmission Electron Microscopy NOV 082000 Investigation of Texture Development in S TI Magnesium Oxide Buffer Layers
}

\author{
Rachel E. Koritala, Michael P. Chudzik, Zhiping Luo, Dean J. Miller, Carl R. Kannewurf, and \\ Uthamalingam Balachandran
}

\begin{abstract}
Biaxially textured magnesium oxide (MgO) buffer layers were grown by inclined substrate deposition and examined before $\mathrm{YBa}_{2} \mathrm{Cu}_{3} \mathrm{O}_{7-\mathrm{x}}$ deposition to optimize their texture. Transmission electron microscopy of buffer layers in both cross-sectional and plan view was used to investigate film microstructure and texture development as a function of deposition thickness $(0.05-3 \mu \mathrm{m})$ and substrate inclination angle $\left(0-55^{\circ}\right.$ from the substrate normal). It was determined that the combined effects of preferential growth of the $\{200\}$ equilibrium crystal habit of $\mathrm{MgO}$ and shadowing by columnar grains led to the development of off-axis (200) -textured films.
\end{abstract}

Index Terms- buffer layers, electron microscopy, inclined substrate deposition, superconducting films.

\section{INTRODUCTION}

$\sigma_{0}$ IGNIFICANT improvement in the critical current densities of $\mathrm{YBa}_{2} \mathrm{Cu}_{3} \mathrm{O}_{7-\mathrm{x}}(\mathrm{YBCO})$ thin films on flexible metal substrates has been obtained by depositing the films on biaxially textured buffer layers [1]. Although both magnesium oxide (MgO) and yttria-stabilized zirconia (YSZ) have been used as buffer layers, $\mathrm{MgO}$ has the advantage of developing texture at a faster rate than YSZ [2]. In addition, $\mathrm{MgO}$ deposited by inclined-substrate deposition (ISD) can be grown on randomly oriented metal substrates at high deposition rates [3].

The critical current density of YBCO films has been shown to degrade as the in-plane misalignment of YBCO grains increases [4]. Because YBCO texture is defined by the texture of the buffer layer on which it is deposited, it is desirable to optimize the texture of the buffer layer. In this study, transmission electron microscopy (TEM) was used to

Manuscript received September 18, 2000.

This work was supported in part by the U.S. Department of Energy (DOE), Office of Energy Efficiency and Renewable Energy, as part of a DOE program to develop electric power technology, under Contract W-31109-Eng-38.

R. E. Koritala and U. Balachandran are with Argonne National Laboratory, Energy Technology Division, Argonne, IL 60439 USA.

M. P. Chudzik was with Argonne National Laboratory, Energy Technology Division, Argonne, IL 60439 USA and Department of Electrical and Computer Engineering, Northwestern University, Evanston, IL 60208 USA. He is now with IBM Microelectronics, Semiconductor R\&D Center, Hopewell Junction, NY 12533 USA.

Z. P. Luo and D. J. Miller are with Argonne National Laboratory, Materials Science Division, Argonne, IL 60439 USA.

C. R. Kannewurf is with Department of Electrical and Computer Engineering, Northwestem University, Evanston, IL 60208 USA. investigate the microstructure and texture of $\mathrm{MgO}$ buffer layers deposited by ISD to optimize their texture for subsequent YBCO deposition. Based on the results, a model for texture development is proposed.

\section{EXPERIMENTAL}

$\mathrm{MgO}$ was deposited on (100) silicon and metal substrates by electron beam evaporation. A schematic representation of the deposition geometry is shown elsewhere [5]. Substrates were inclined at an angle $\alpha$ with respect to the vapor source, with $\alpha$ ranging from 0 to $55^{\circ}$. The film thickness was kept constant at $2.5 \mu \mathrm{m}$ while $\alpha$ was varied. Oxygen was introduced into the deposition chamber to ensure oxygen stoichiometry. The pressure during evaporation was $2 \times 10^{-5}$ torr. Deposition was performed at room temperature at rates of 2.5 to $100 \AA /$ second, with the thickness of the resulting films ranging from $500 \AA$ to $5 \mu \mathrm{m}$.

TEM was performed in a Philips CM30 microscope operated at $300 \mathrm{keV}$. Plan-view TEM specimens were prepared from films deposited on silicon substrates by mechanical grinding from the undeposited side, followed by dimpling. Specimens were then ion-milled from the undeposited side to perforation with a precision ion polishing system (GATAN). Cross-sectional TEM specimens were prepared in a similar manner by gluing two sample pieces together, with deposited sides facing. Specimens were then ground on both sides, dimpled from one side, and ion-milled from both sides.

\section{RESULTS}

A cross-sectional TEM bright-field image and corresponding selected area diffraction (SAD) pattern for $\mathrm{MgO}$ deposited at normal incidence $\left(\alpha=0^{\circ}\right)$ are shown in Fig. 1. As seen in the SAD pattern, the film exhibits (111) out-of-plane texture. However, when the same sample is observed in plan view, as shown in Fig. 2, no in-plane texture is seen, as demonstrated by the polycrystalline SAD pattern. Because in-plane texture is required for coated-conductor applications, films grown at this angle are not useful.

Increasing the inclination angle $\alpha$ significantly improves the in-plane texture of the films. This improvement is demonstrated in the SAD pattern in Fig. 3, which corresponds to a plan-view image of a sample deposited at $\alpha=55^{\circ}$. Results were confirmed by $x$-ray diffraction [5]. The texture also improves with increasing film thickness. Fig. 4 is a 

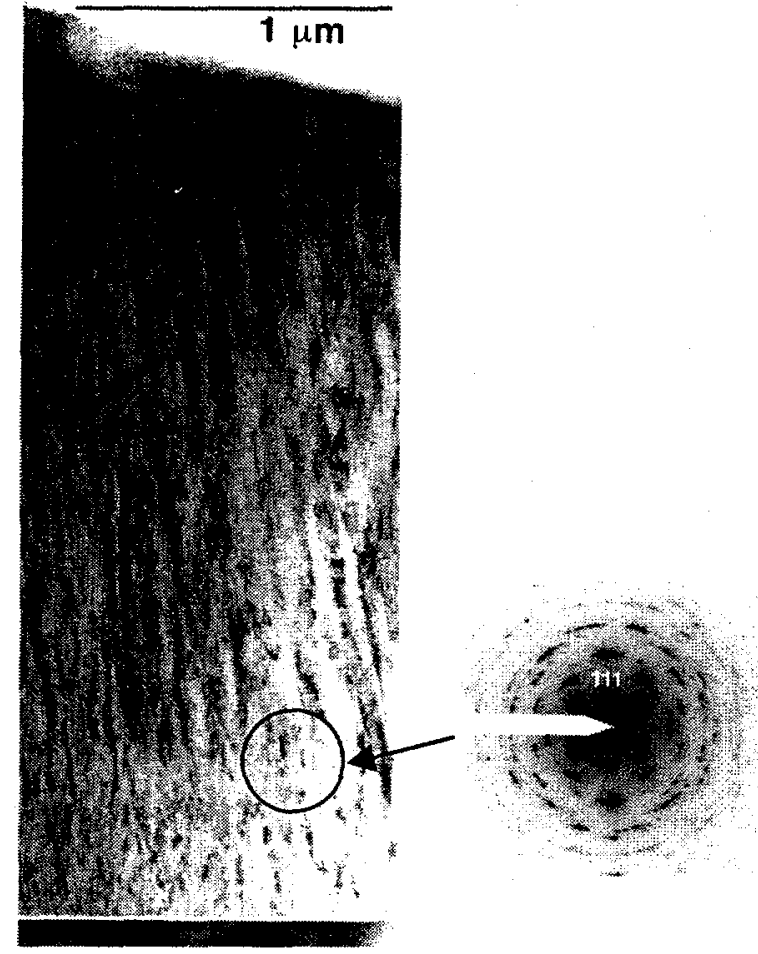

Fig. 1. Cross-sectional TEM image and SAD pattern of MgO film deposited at $\alpha=0^{\circ}$.

cross-sectional TEM image of MgO deposited at $\alpha=55^{\circ}$. $\mathrm{SAD}$ patterns from various regions throughout the thickness are also shown and clearly indicate an improvement in texture as the film grows. The pattern from the region immediately above the interface demonstrates polycrystalline orientation. The film becomes well-textured at a thickness of $\approx 1 \mu \mathrm{m}$.

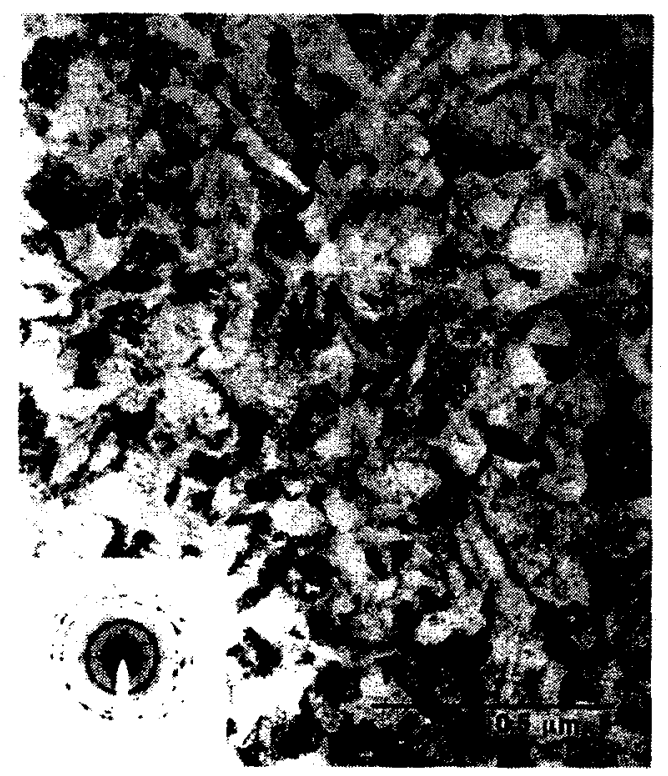

Fig. 2. Plan-view TEM image and SAD pattern of $\mathrm{MgO}$ film deposited at $\alpha=0^{\circ}$.
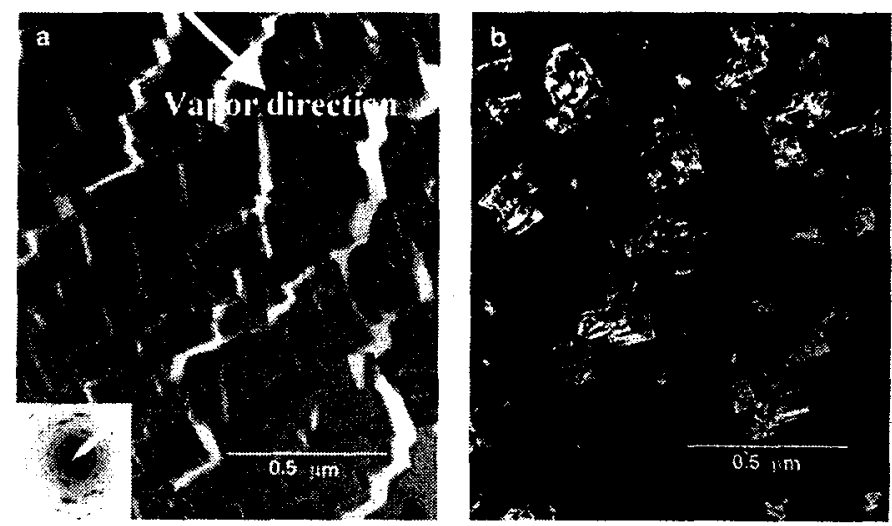

Fig. 3. Plan view (a) bright field and (b) dark field images of $\mathrm{MgO}$ film deposited at $\alpha=55^{\circ}$, and corresponding SAD pattern.

Further confirmation of this improvement is provided by examining films of various thicknesses. Fig. 5 shows planview images and SAD patterns of films that are $500 \AA$ and 3 $\mu \mathrm{m}$ thick, both deposited at $\alpha=55^{\circ}$. The grains in the thinner film exhibit polycrystalline orientation, whereas the thicker film exhibits a well-defined texture.

Fig. 5 also demonstrates the variation in grain morphology as the film grows. Grains in the $500-\AA$ film are small $(\approx 20$ $\mathrm{nm}$ ), nearly equiaxed, and dense. In the $3-\mu \mathrm{m}$ film, on the other hand, grains are larger $(\approx 100-200 \mathrm{~nm})$ and exhibit cubic morphology. The cubic morphology of the thicker films can be seen more clearly in the dark field image shown in Fig. 3. The grain morphology can also be observed in cross-section. As shown in Figs. 1 and 4, films grown at any angle exhibit a distinct columnar structure. The tops of the columns are

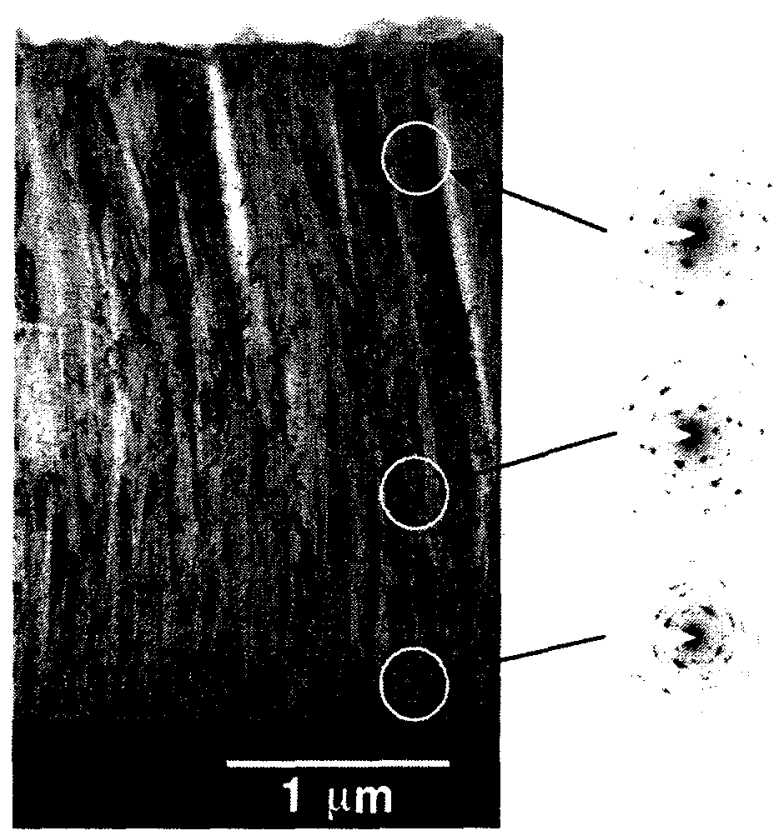

Fig. 4. Cross-sectional TEM image and SAD patterns at various thicknesses in $\mathrm{MgO}$ film deposited at $\alpha=55^{\circ}$. Film viewed perpendicular to vapor direction. 

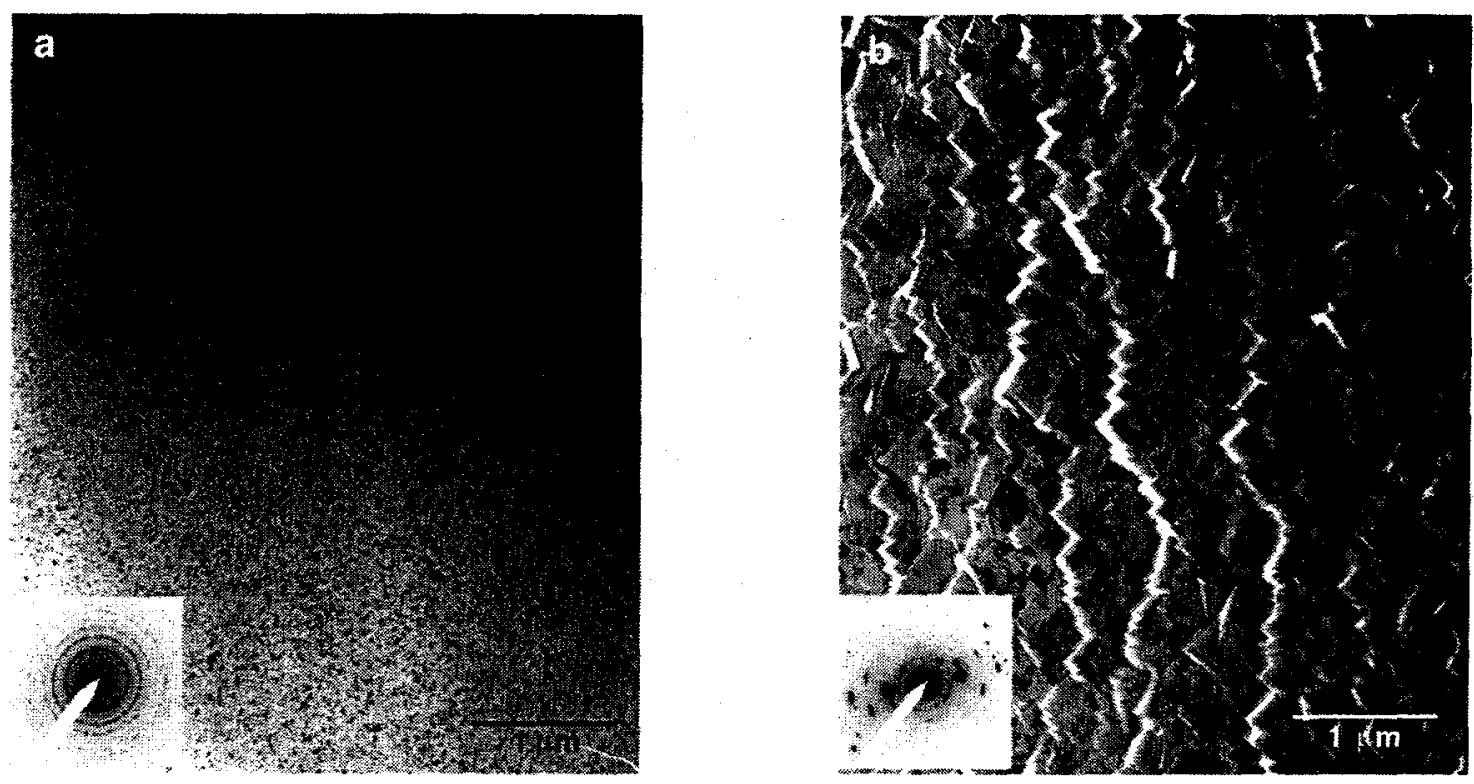

Fig. 5. Plan view TEM images and SAD patterns of (a) 500- $\AA$ thick and (b) 3- $\mu \mathrm{m}$ thick MgO films

truncated by (200) planes, as shown in the SAD pattern and corresponding image in Fig. 6.

Finally, the microstructure varies with respect to the vapor direction. Fig. 7 is a cross-sectional TEM image of a film grown at $\alpha=55^{\circ}$ and observed in a direction parallel to the vapor direction. It can be seen that columns are dense throughout the entire film thickness. This is in contrast to the same film observed perpendicular to the vapor direction, shown in Fig. 4. In this image, beyond a film thickness of $\approx 1$ $\mu \mathrm{m}$, gaps can be seen between columns. Jagged gaps were also observed between rows of grains in the plan view image of the $3-\mu \mathrm{m}$ film in Fig. 5.

\section{DISCUSSION}

Film morphology and texture evolve in the manner described above because of the growth mechanisms during ISD of $\mathrm{MgO}$. It has been demonstrated [6] that in-plane texture during polycrystalline film growth develops through a combination of fast growth along a certain crystallographic direction and self-shadowing that occurs when deposition is

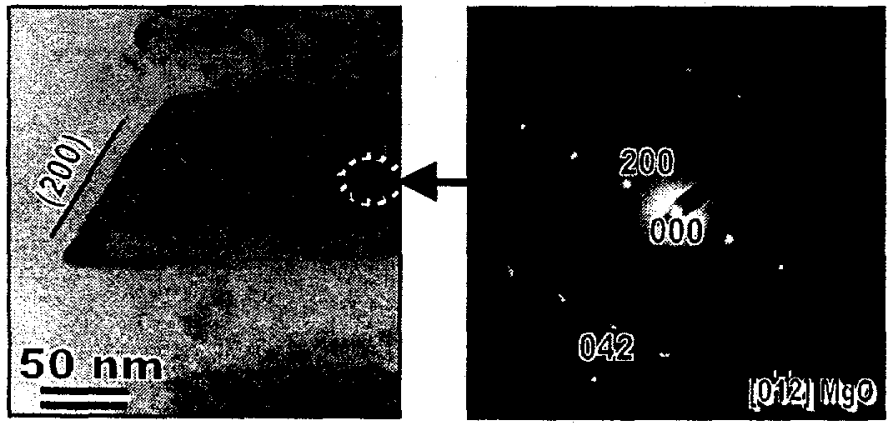

Fig. 6. Facet on top of $\mathrm{MgO}$ column in film deposited at $\alpha=55^{\circ}$. The corresponding SAD pattern shows that the column face is a (200) plane. at an inclined angle. In the case of $\mathrm{MgO}$, the fast-growthplane is $\{200\}$ [7]. Because maximizing the (200) faces decreases the surface free energy, the $\{200\}$ plane is also the equilibrium crystal habit, as confirmed by the cubic morphology exhibited in the $\mathrm{MgO}$ film in Fig. 3. With deposition at an inclined angle, the $\{200\}$ plane rotates toward the vapor as growth occurs so that the (200) surface area is maximized. This effect has been confirmed with $\mathrm{x}$-ray

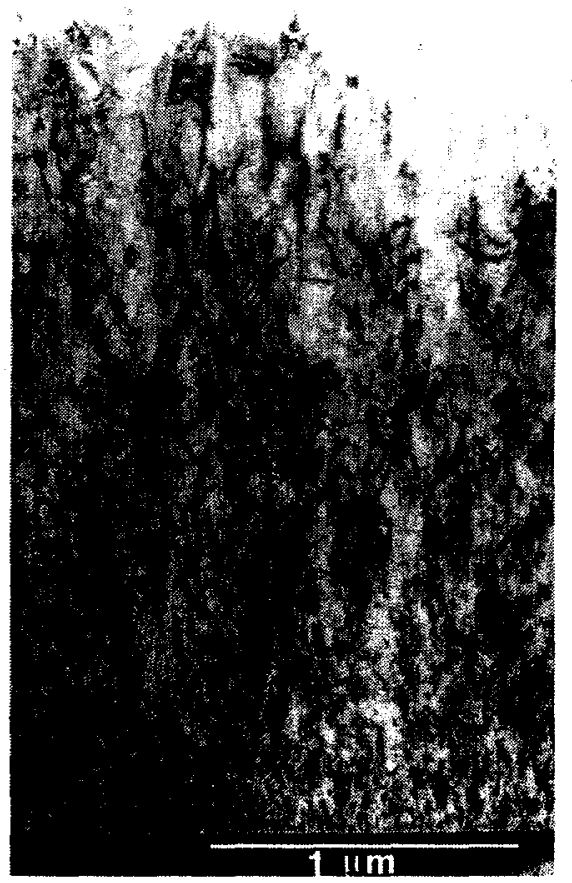

Fig. 7. Cross-sectional TEM image of MgO film deposited at $\alpha=55^{\circ}$, viewed parallel to vapor direction. 


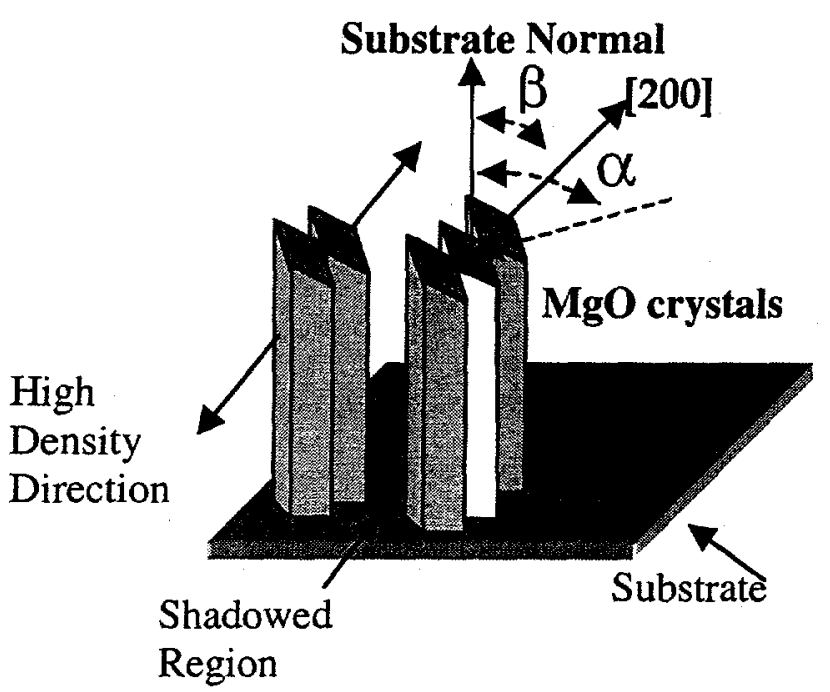

Fig. 8. Schematic representation of shadowing effect, where $\alpha$ is the angle between substrate normal and vapor direction and $\beta$ is the orientation angle (degree to which [200] is inclined toward vapor flux).

analysis [5]. A schematic representation of grain morphology with respect to the vapor direction is shown in Fig. 8.

Because $\{200\}$ planes grow faster than others, columns with this orientation eventually grow taller than columns with other orientations. This leads to shadowing of slowergrowing grains, which eventually become extinct as they intersect the column walls of taller grains. Several micrographs demonstrate the shadowing effect. The planview image in Fig. 3 and cross-sectional image in Fig. 4 show gaps between rows of grains, where shadowed grains have become extinct. Fig. 4 also shows the region near the substrate/MgO interface where the grains are denser and smaller because columns of various orientations are present and $\{200\}$-oriented grains have not grown tall enough to shadow others. This effect is confirmed by the small, dense, randomly oriented grains in the plan-view image of the 500$\AA$ film in Fig. 5. Finally, the cross-sectional image viewed parallel to the vapor direction in Fig. 7 can be compared with the same film viewed perpendicular to the vapor direction in Fig. 4 to demonstrate the difference in density with respect to vapor direction, as illustrated in Fig. 8.

Both the $\{200\}$ equilibrium habit and the shadowing effect combine to improve texture as the film grows. This phenomenon is depicted in the SAD patterns in Fig. 4.

\section{CONCLUSIONS}

TEM was used to examine $\mathrm{MgO}$ buffer layers that were grown by ISD. The in-plane texture improves both with increasing inclination angle $\alpha$ and as the film thickness increases. Films examined in cross-section show columnar grain morphology, with tops truncated by (200) planes. Gaps were observed between columns when viewed perpendicular to the vapor direction, but not when viewed parallel. Films exhibit the observed morphology and texture because of the combined effects of shadowing by columnar grains and the preferential growth of the $\{200\}$ equilibrium crystal habit of MgO.

\section{ACKNOWLEDGMENT}

TEM analysis was performed in the Electron Microscopy Center for Materials Research at Argonne National Laboratory.

\section{REFERENCES}

[1] S. R. Foltyn, P. N. Ardent, P. C. Dowden, R. F. Depaula, J. R. Groves,

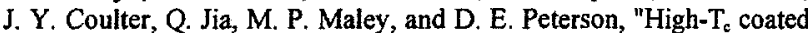
conductors - performances of meter-long YBCO/BAD flexible tapes," IEEE Trans. on Appl. Supercond., vol. 9, pp. 1519-1522, June 1999.

[2] C. P. Wang, K. B. Do, M. R. Beasley, T. Geballe, and R. Hammond, "Deposition of in-plane textured $\mathrm{MgO}$ on amorphous $\mathrm{Si}_{3} \mathrm{~N}_{4}$ substrates by ion-beam-assisted deposition and comparisons with ion-beamassisted deposited yttria-stabilized-zirconia," Appl. Phys. Lett., vol. 71, pp. 2955-2957, November 1997.

[3] M. Bauer, R. Semerad, and H. Kinder, "YBCO films on metal substrates with biaxially aligned MgO buffer layers," IEEE Trans. on Appl. Supercond., vol. 9, pp.1502-1505, June 1999.

[4] D. Dimos, P. Chaudhari, J. Mannhart, and F. K. LeGoues, "Orientation dependence of grain-boundary critical currents in $\mathrm{YBa}_{2} \mathrm{Cu}_{3} \mathrm{O}_{7-\delta}$ bicrystals," Phys. Rev. Lett., vol. 61, pp. 219-222, July 1988.

[5] M. P. Chudzik, R. E. Koritala, Z. P. Luo, D. J. Miller, U. Balachandran, and $C$. R. Kannewurf, "Mechanism and processing dependence of biaxial texture development in magnesium oxide thin films grown by inclined-substrate deposition," IEEE Trans. on Appl. Supercond., submitted for publication.

[6] O. P. Karpenko, J. C. Bilello, and S. M. Yalisove, "Growth anisotropy and self-shadowing: A model for the development of in-plane texture during polycrystalline thin-film growth," J. Appl. Phys., vol. 82, pp. 1397-1403, August 1997.

[7] A. F. Moodie and C. E. Warble, "Electron microscopic investigation of $\mathrm{MgO}$ morphology and surfaces," J. Crystal Growth, vol. 10, pp. 26-38, 1971. 